\title{
MONOGRÁFICO
}

\section{Las didácticas específicas en tiempos de COVID-19}

\author{
Ma del Mar SUÁREZ \\ Universitat de Barcelona \\ mmsuarez@ub.edu \\ https://orcid.org/oooo-0oo2-1741-7596
}

\begin{abstract}
Cuando el consejo de redacción de esta revista acordó publicar una sección monográfica sobre los efectos de la COVID-19 en esta revista, ni mucho menos creíamos ser para nada originales. De hecho, se cuentan por decenas los números especiales y libros monográficos sobre el tema. La rapidez en que proliferaron las solicitudes de contribuciones y las peticiones de rellenar cuestionarios en línea se hicieron tan virales que algunos, y aquí me incluyo, llegamos al hartazgo. Esa viralidad y esa prisa también pueden conllevar, naturalmente, un descenso en la calidad de los trabajos. En nuestro caso, nos lo tomamos con la calma. La fecha límite de envío se planeó para el 30 de junio del 2021. Aquí una servidora temía que para cuando saliera el número publicado, el tema ya estuviera más que trillado (que un poco sí) o, peor aún, démodé (para la revista, se entiende). Por suerte para la revista, y por desgracia para el resto de la humanidad (iahí es nada!), esto último no es así. Ahí seguimos, con estadísticas de los afectados por la COVID-19 en los periódicos y telediarios. Con mucha menos frecuencia y sin ya tanta insistencia en las víctimas mortales, esto es así. Seguimos también, por suerte, con los porcentajes de vacunados y gratas noticias acerca de la menor gravedad con la que nos ataca el dichoso bicho.
\end{abstract}

EI SARS-CoV-2 y sus mil variantes, además de acercarnos a ese gran desconocido por algunos que es el alfabeto griego, ha hecho que, primero de la noche a la mañana, y luego ya con más margen de reacción, se modificara nuestro día a día. Este hecho ha facilitado también que la revisión de los artículos publicados en esta sección monográfica que nos ocupa gocen también, en cierto modo, de una perspectiva más, digámosle desafectada, facilitada por el paso del tiempo y el reposo de las ideas. No es así, no obstante, en el editorial que le precede ("Esto no lo estudié en la carrera"), de Gemma Barrera Gil. Escrito en enero de 2021, es un editorial lleno de carga emotiva y sentimental, lo que nos orgullece profundamente. En este texto expresa precisamente esa incertidumbre, ese cambio en la normalidad de las aulas, ese estar pero no poder estar al cien por cien, que no llega a ser un malestar pero tampoco un bienestar pleno. Esa nueva normalidad incómoda que todos estamos experimentando y que hace que inevitablemente nos pongamos en la piel de no solo los profesores, sino también de los estudiantes, esos "campeones" abocados no solo a los intríngulis de lo que es crecer y madurar, que no es poco, sino también a las restricciones y normas cambiantes según la 
estadística y el gestor de turno. Nada que, en principio, no puedan salvar las tecnologías -en la teoría, damas y caballeros-. Que sí, que las tecnologías nos tienen a muchos conectadísimos, pero también es lo que, a la vez, nos distancia. iQué ironía! Estando sin estar. Y sin saber hasta cuándo.

Enceta la sección monográfica el trabajo de Amando López Valero y sus colegas de la Universidad de Murcia titulado "Competencia comunicativa, didáctica de la lengua y adaptación sociosanitaria: ¿Una aceleración de la cuarta revolución industrial?". Si bien es un trabajo dentro del marco de la Didáctica de la Lengua y la Literatura, tuvimos claro desde el principio que era este, y no otro, el que debía abrir este especial. La razón es muy simple: sin querer, nos enseña cómo los cambios en la comunicación diaria en tiempos de COVID-19 trascienden el aula y nos afectan en nuestro día a día. No podíamos estar tampoco más de acuerdo con su planteamiento: la paradoja de estar conectados permanentemente incide en un mayor aislamiento. Ciertamente, el uso diario de la tecnología implica también cambios en nuestro uso de la lengua y en el enfoque que podemos adoptar para su enseñanza, optando, por ejemplo, por un enfoque por competencias, incluyendo las digitales y las informacionales, aunque estas pueden conllevar, a su pesar, una disminución del desarrollo léxico del estudiante por la pasividad en la recepción de la información. Más por menos.

Sin quererlo, nos encontramos con otros tres artículos que tienen como eje central el lenguaje. Seamos honestos: las tecnologías, así como lidiar con las distancias, son transversales y las tenemos más o menos exploradas. Cada día sale una plataforma nueva, más mona que la anterior, con ese detalle que la hace especial, pero que tampoco va a revolucionar el mundo de la enseñanza. Eso ya lo hicieron las MOOCs en su momento y no por su virtualidad, sino por su generosidad. Ni la realidad virtual parece acabar de encontrar su lugar en la enseñanza. Lo que sí nos faltaba por explorar era un contexto pandémico que hiciera que no nos quedara más remedio que optar por la tecnología como principal modus operandi y casi que vivendi.

Partiendo de que ya bastante trabajo nos cuesta enseñar a escribir en el aula, no digamos que virtualmente, Cristopher David Herrera Navas, Gabriela Ibeth Saltos Piguave y Edgar Efraín Obaco Soto presentan el manuscrito: "Producción de textos mediante entornos educativos virtuales del software Ardora: Una experiencia de aplicación en pandemia". En él, los resultados indican que, efectivamente, usar el software Ardora mejora la producción de textos escritos en la población estudiada. No obstante, y volvemos a lo mismo, los autores no encuentran que Ardora sea la herramienta mágica con superpoderes que solucione todas las habilidades de escritura de los estudiantes, entre las que se incluyen la acentuación, la puntuación, la coherencia y la cohesión.

La virtualidad afecta también sobremanera aquellas asignaturas con un gran componente práctico o concebidas para un desarrollo presencial, en lo que podríamos llamar "la vida real". Este es el caso de los manuscritos de Paloma López-Zurita, en primer lugar, y de Jessica Pérez-Luzardo, para continuar. En el primer caso, en "The impact of COVID-19 in an ESP course: An unforeseen adaptation to distance teaching" ("El impacto de la COVID-19 en un curso de Inglés con Fines Específicos: Una adaptación imprevista a la enseñanza no presencial"), la autora presenta un estudio del caso de una asignatura de Inglés con Fines Específicos cuyo enfoque, por la situación pandémica, se tuvo que cambiar forzosamente, convirtiéndola así en una asignatura eminentemente práctica. El cuestionario administrado a los estudiantes señala 
los aspectos que consideran que deberían mejorarse en esta asignatura en una situación de emergencia. No por ello se presenta una experiencia deficiente. Que levante la mano a quién le salió todo bien a la primera en pleno auge de la pandemia. Lo que hace este estudio especial es ver cómo los estudiantes comparan los contextos presenciales con la nueva situación. El preferido en el sentido interaccional vuelve a ser, oh sorpresa, el entorno presencial. Por algo será. Pero sí que es verdad que esta situación nos ha conducido a un desarrollo forzoso de nuestra competencia digital, formación que ha llegado para quedarse.

El estudio de caso de Jessica Pérez-Luzardo, "Universidad presencial confinada: Percepción de los estudiantes de Interpretación de la docencia presencial, semipresencial y no presencial durante la pandemia", nos introduce también en la afectación de una asignatura también basada en la comunicación, para ser más específicos, en la asignatura de Interpretación. Tras presentar la adaptación curricular correspondiente, a través de un cuestionario Pérez-Luzardo nos transmite las opiniones de los estudiantes al comparar entornos presenciales, semipresenciales y virtuales. Nuevamente podríamos decir "and the winner is..." en cuestiones de interacción y de facilidad y calidad de la conexión a internet, pero se observan también las ventajas de los entornos virtuales en cuanto a evitar desplazamientos y se aprecia la mayor flexibilidad que aportan.

Cierra el bloque dedicado a los trabajos que versan sobre asignaturas donde el desempeño comunicativo es central el estudio cualitativo de Melisa Soledad Gigena y Azahara Cuesta García "Estaciones de aprendizaje en una clase de ELE en línea para adultos: percepciones sobre sus facilidades y dificultades". Este estudio se centra en una experiencia ya de por sí novedosa: el aprendizaje por estaciones en dos grupos en línea. Las catorce entrevistas con estudiantes, los cuatro informes de observación focalizada de profesores observadores y un diario docente revelan que si algo común podemos sonsacar de este y de los estudios presentados en el resto de la sección monográfica es el amplio abanico de posibilidades que presentan los entornos virtuales y el esfuerzo por la atención personalizada dirigida al alumno. Ahora bien, medir la energía que esta atención supone, así como la gestión del tiempo y el tipo de instrucciones no son temas baladís en un entorno virtual. Por suerte, además de la propia percepción del profesor, tenemos a los estudiantes que nos pueden hacer notar qué aspectos mejorar en nuestra práctica docente en dichos entornos.

Clausura el monográfico un trabajo que se aleja del área de especialidad de los anteriores, pero que no por ello deja de presentar retos similares. Se trata de "El trabajo práctico de laboratorio en clase de Ciencias Naturales durante la pandemia: Experiencias en Argentina y Colombia", artículo de Fanny Angulo-Delgado, Alejandro Calle y Carlos Soto-Lombana, de la Universidad de Antioquia (Colombia), junto a Erica Zorrilla y Claudia Alejandra Mazzitelli, ambas de la Universidad Nacional de San Juan (Argentina). En él se aborda la adaptación de los trabajos prácticos de laboratorio a un entorno virtual en dos países latinoamericanos distintos. Aunque sus resultados indican que los profesores fueron capaces de adaptar sus estrategias de enseñanza, apoyándose en los recursos digitales disponibles y replanteando los objetivos iniciales, ese esfuerzo se vio limitado inevitablemente por la virtualidad, a la que los estudiantes de zonas menos agraciadas tenían acceso limitado y, por tanto, sus oportunidades de aprendizaje se vieron también menguadas.

Vislumbramos a través de los trabajos incluidos en esta sección monográfica, y también en los trabajos que finalmente no se aceptaron, que los aspectos comunicativos no solo son 
transversales, sino cruciales en una situación pandémica donde la docencia pasó de la noche a la mañana a un contexto virtual en su totalidad. Con ello, también nos replanteamos cómo pueden haber pasado tantos años sin haber superado aún la famosa brecha digital, ya sea competencial, ya sea por las dificultades de acceso a internet. No todos tenemos las mismas oportunidades, y menos aún en pandemia. Hace falta hacer algo al respecto a la voz de ya. 